\title{
Issues in Efficiency for Distributed and Networking Systems
}

\author{
Guest Editor: Ju Yeon Jo
}

This special issue is dedicated to the $15^{\text {th }}$ annual International Conference on Software Engineering, Artificial Intelligence, Networking and Parallel/Distributed Computing (SNPD 2014) held on June 30-July 2, 2014 in Las Vegas, USA. SNPD brings together researchers, scientists, engineers, industry practitioners, and students to discuss, encourage and exchange new ideas, research results, and experiences on all aspects of computer and information science. This special issue captures seven of the conference's most promising papers which have been revised and extended for this publication. We impatiently await the important contributions that we know these authors will bring to the field.

The first paper by Ayaz ul Hassan Khan, Mayez Al-Mouhamed, Allam Fatayer, Anas Almousa, Abdulrahman Baqais and Mohammed Assayony is entitled "Padding Free Bank Conflict Resolution for CUDA-Based Matrix Transpose Algorithm". The authors propose two matrix transpose algorithms to alleviate the aforementioned issues of ensuring coalesced access and conflict free bank access. The proposed algorithms have comparable execution times with the NVIDIA SDK bank conflict - free matrix transpose implementation. The main advantage of proposed algorithms is that they eliminate bank conflicts while allocating shared memory exactly equal to the tile size ( $\mathrm{T} \times \mathrm{T}$ ) of the problem space.

The second paper by Tahir Jameel and Mengxiang Lin is entitled "Test Image Generation using Segmental Symbolic Evaluation". The authors present a technique that is the first of its type to generate test images based on symbolic evaluation of program under test. The idea is based on the fact that, neighboring image operations are applied by selecting a segment of image pixels called a window, and iterated by sliding window over entire image. They have tested the proposed scheme on different programs and the results show that test images are successfully generated for each path to ensure the path coverage of the program under test and identifying infeasible paths.

The third paper by Lifford McLauchlan, Soumya Saha and Rajab Challoo is entitled "Comparative Study of SWST (Simple Weighted Spanning Tree) and EAST (Energy Aware Spanning Tree)". The authors present a comparative study of the SWST and EAST algorithms conducted to evaluate the performance of the two algorithms. From the MATLAB simulations it was observed that the EAST protocol generally performs better than the SWST algorithms in delivering messages to the sink node.

In the fourth paper by Mohammad Fal Sadikin and Marcel Kyas is entitled "Efficient Security and Privacy Protection for Emerging Smart RFID Communications". The authors present IMAKA-Tate: Identity protection, Mutual Authentication and Key Agreement using Tate pairing of Identity-based Encryption method. It is designed to tackle various challenges in the constrained nature of RFID applications by applying a light-weight cryptographic method with advanced-level 128 bit security protection.

In the fifth paper by Takashi Kuremoto*, Takuhiro Otani, Shingo Mabu, Masanao Obayashi and Kunikazu Kobayashi is entitled "One-D-R-A-G-SOM and its Application to a Hand Shape Instruction Learning System". The authors propose a novel self-organizing map (SOM) named "One-D-R-A-G-SOM", which is a kind of one dimensional ring type growing SOM using asymmetric neighborhood function. Experiment results showed the effectiveness of the novel system comparing with systems using the conventional SOMs.

In the sixth paper by Nermin Kajtazovic, Christopher Preschern, Andrea Holler, and Christian Kreiner is entitled "On Design-time Modeling and Verification of Safety-critical Component-based Systems". The authors present a novel approach for design-time modeling and verification of safety-critical systems, based on data semantics of

Published by Atlantis Press

Copyright: the authors 
components. They describe the composition, i.e., the systems design, and the underlying properties of components as a Constraint Satisfaction Problem (CSP) and perform the verification by solving that problem.

In the seventh paper by Lifford McLauchlan, Soumya Saha, Claudio Montiel and Rajab Challoo is entitled “Comparative Study of Various Wireless Sensor Network (WSN) Topology Construction Protocols". The authors propose two new load balancing TC protocols, SWST (Simple Weighted Spanning Tree), EAST (Energy Aware Spanning Tree) as well as three well known TC protocols, Simple Tree, Random Nearest Neighbor Tree (Random NNT) and Euclidean Minimum Spanning Tree (Euclidian MST), are studied using MATLAB and TC protocols such as A3 (A tree), A3 Coverage, Connected Dominating Set under Rule K (CDS Rule K), Energy Efficient Connected Dominating Set (EECDS), Simple Tree and K Neighbor (KNeigh) Tree, are simulated using Atarraya. Comparisons are performed between many of the TC protocols.

We wish to express their gratitude to the authors for their contributions, to the reviewers for their cooperation and constructive advices that led to further improvements of the articles. We also wish to express our thanks to Bertil Folliot and Juhnyoung Lee, Editors-in-Chief of the Journal, for accepting to publish this Special Issue and for their help throughout the publication process.

Ju Yeon Jo

University of Nevada-Las Vegas

Las Vegas, Nevada, USA

Email: juyeon.jo@unlv.edu 